\title{
Equivalence of Topological and Scattering Approaches to Quantum Pumping
}

\section{Journal Article}

\section{Author(s):}

Bräunlich, G.; Graf, G. M.; Ortelli, G.

Publication date:

2010

Permanent link:

https://doi.org/10.3929/ethz-b-000017256

\section{Rights / license:}

In Copyright - Non-Commercial Use Permitted

\section{Originally published in:}

Communications in Mathematical Physics 295(1), https://doi.org/10.1007/s00220-009-0983-1 


\title{
Equivalence of Topological and Scattering Approaches to Quantum Pumping
}

\author{
G. Bräunlich, G. M. Graf, G. Ortelli \\ Theoretische Physik, ETH-Zürich, CH-8093 Zürich, \\ Switzerland. E-mail: gmgraf@itp.phys.ethz.ch
}

Received: 28 February 2009 / Accepted: 2 October 2009

Published online: 27 December 2009 - (C) Springer-Verlag 2009

\begin{abstract}
The Schrödinger equation with a potential periodically varying in time is used to model adiabatic quantum pumps. The systems considered may be either infinitely extended and gapped or finite and connected to gapless leads. Correspondingly, two descriptions of the transported charge, one relating to a Chern number and the other to a scattering matrix, have been available for some time. Here we generalize the first one and establish its equivalence to the second.
\end{abstract}

\section{Introduction}

Quantum pumps are driven devices connected to leads kept at a same voltage. Two descriptions of charge transport are available for pumps depending on time periodically and adiabatically. One has been proposed by Thouless [19] (see also [16]), the other by Büttiker et al. [7] (see also [6]). We shall refer to them as the topological, resp. the scattering approaches and denote by $\left\langle Q_{T}\right\rangle$, resp. $\left\langle Q_{B P T}\right\rangle$ the charges transported during a cycle. Each one depends on a different idealization of the devices. In the first proposal the model is a non-interacting Fermi gas, infinitely extended in one dimension with the Fermi energy lying in a gap. The charge transported within a period appears as a Chern number, indicating that it is quantized. In the second approach the device is viewed as a compact object connected to leads containing free, gapless Fermi gases. Here, the transported charge is expressed in terms of the scattering matrix at Fermi energy and is quantized in special cases only.

At first sight charge transport is accounted for in rather different, if not opposing, ways: The spatial extent of the two devices is infinite, resp. finite, reflecting a microscopic, resp. macroscopic, perspective; more strikingly, in the first case transport is attributed to energies way below the Fermi energy, which lies in a spectral gap, while in the second the scattering matrix matters only at Fermi energy. In physical terms, the first description applies to insulators, the second to conductors, at least seemingly so. 
Yet, the two points of view are mathematically related. This has been shown in [10] for the simpler case of a single channel, modeled as a real line, and of a potential which is periodic also in space. A comparison becomes possible after truncating the potential to finitely many periods, while the rest of the line gives rise to the leads. Then the spectral gap closes and the model becomes amenable to the scattering approach. There, the conditions for quantized transport are attained in the limit of many periods, and quantitative agreement between the two approaches was established.

In this article we generalize the equivalence result in two ways, thereby extending it to the natural setting of both approaches. First, the requirement of spatial periodicity [19] is dropped. Such a situation was considered in [16], though by approximating a general (e.g. quasi-periodic) potential by a sequence of periodic ones with increasing periods. Only the approximants were associated to fiber bundles, based on the corresponding Brillouin zones. Here we propose a bundle and hence a Chern number applying directly to the infinite, non-periodic system. Second, we extend the correspondence [10] to a multi-channel setting.

As far as we know, the earliest statement concerning the equivalence is found in [8], though only for a particular, exactly solvable, periodic, tight binding Hamiltonian. On more general terms we note that, albeit the topological approach predates the scattering approach, several ideas underlying the equivalence can be traced back to [19]. Experimental work which is thematically related is described e.g. in $[5,12,17]$.

In Sect. 2 we state the results for charge transport based on the two approaches separately, and formulate the comparison, which is the main result, as Theorem 2. In Sect. 3 we describe the relevant fiber bundle, while Sect. 4 is devoted to proofs. An Appendix provides a result in adiabatic perturbation theory.

\section{Main Results}

We begin by describing the topological approach [19] in the case of $n$ channels. The Hamiltonian, acting on $L^{2}\left(\mathbb{R}_{x}, \mathbb{C}^{n}\right)$, is

$$
H(s)=-\frac{d^{2}}{d x^{2}}+V(x, s),
$$

where the potential $V=V(x, s)$ takes values in the $n \times n$ matrices, $M_{n}(\mathbb{C})$, is Hermitian, $V=V^{*}$, and periodic in time, $V(x, s+2 \pi)=V(x, s)$. For simplicity, let $V(\cdot, s) \in L^{\infty}\left(\mathbb{R}_{x}, M_{n}(\mathbb{C})\right)$ with $C^{1}$-dependence on $s \in S^{1}:=\mathbb{R} / 2 \pi \mathbb{Z}$. Then, for any $z \in \rho(H(s))$ in the resolvent set, the Schrödinger equation $H(s) \varphi=z \varphi$ is in the limit-point case at $x=+\infty$ (see $[14$ or 9,13$]$ ), meaning that as an ordinary differential equation it has $n$ linearly independent solutions which are square-integrable at $x=+\infty$. We may thus introduce a family of sets, parametrized by $z \in \rho(H(s))$ and $s \in S^{1}$, consisting of matrix-valued solutions $\psi(x) \in M_{n}(\mathbb{C})$ of the Schrödinger equation

$$
-\psi^{\prime \prime}(x)+V(x, s) \psi(x)=z \psi(x),
$$

which are regular in the sense that for any $x \in \mathbb{R}$,

$$
\psi(x) a=0, \psi^{\prime}(x) a=0 \Rightarrow a=0, \quad\left(a \in \mathbb{C}^{n}\right) .
$$

It is:

$$
S_{(z, s)}^{+}=\left\{\psi_{+} \mid \psi_{+} \text {is a regular solution of }(2), L^{2} \text { at } x=+\infty\right\}
$$


As a matter of fact such solutions tend to zero pointwise as $x \rightarrow+\infty$, together with their first derivatives. Similarly, solutions $\tilde{\psi}(x) \in M_{n}(\mathbb{C})$ of the adjoint equation

$$
-\tilde{\psi}^{\prime \prime}(x)+\tilde{\psi}(x) V(x, s)=z \tilde{\psi}(x)
$$

act on row vectors $a \in \mathbb{C}^{n}$ as $a \tilde{\psi}(x)$, and we set

$$
\tilde{S}_{(z, s)}^{-}=\left\{\tilde{\psi}_{-} \mid \tilde{\psi}_{-} \text {is regular solution of }(5), L^{2} \text { at } x=-\infty\right\} .
$$

For later use we also introduce the families $S_{(z, s)}^{-}, \tilde{S}_{(z, s)}^{+}$of solutions to (2), resp. (5) decaying at the opposite ends. For any two differentiable functions $\psi, \tilde{\psi}: \mathbb{R} \rightarrow M_{n}(\mathbb{C})$ we define the Wronskian

$$
W(\tilde{\psi}, \psi ; x)=\tilde{\psi}(x) \psi^{\prime}(x)-\tilde{\psi}^{\prime}(x) \psi(x) \in M_{n}(\mathbb{C}) .
$$

It is independent of $x$ if $\psi$ and $\tilde{\psi}$ are solutions of (2), resp. of (5), in which case it is simply denoted as $W\left(\tilde{\psi}_{-}, \psi_{+}\right)$. As will also be shown later, $\operatorname{det} W\left(\tilde{\psi}_{-}, \psi_{+}\right) \neq 0$ for $\psi_{+} \in S_{(z, s)}^{+}, \tilde{\psi}_{-} \in \tilde{S}_{(z, s)}^{-}$. We observe that $S_{(z, s)}^{+}$carries a transitive right action of $\mathrm{GL}(n) \ni T$,

$$
\psi_{+}(x) \mapsto \psi_{+}(x) T,
$$

while $\tilde{S}_{(z, s)}^{-}$carries a left action,

$$
\tilde{\psi}_{-}(x) \mapsto T \tilde{\psi}_{-}(x) .
$$

We thus have a bijective relation between $\psi_{+} \in S_{(z, s)}^{+}$and $\tilde{\psi}_{-} \in \tilde{S}_{(z, s)}^{-}$such that

$$
W\left(\tilde{\psi}_{-}, \psi_{+}\right)=1 \text {. }
$$

We assume that the Fermi energy $\mu>0$ lies in a spectral gap at all times $s$ :

$$
\mu \in \rho(H(s)) .
$$

Let $P_{0}(s)$ be the spectral projection of $H(s)$ up to the Fermi energy and $U_{\varepsilon}\left(s, s_{0}\right)$ be the propagator for the non-autonomous Hamiltonian $H(\varepsilon t)$, where $s=\varepsilon t$. In the Appendix we prove, in the smooth case,

$$
U_{\varepsilon}\left(s, s_{0}\right)\left(P_{0}\left(s_{0}\right)+\varepsilon P_{1}\left(s_{0}\right)\right) U_{\varepsilon}\left(s, s_{0}\right)^{*}=P_{0}(s)+\varepsilon P_{1}(s)+O\left(\varepsilon^{2}\right), \quad(\varepsilon \rightarrow 0)
$$

with

$$
P_{1}(s)=-\frac{1}{2 \pi} \oint_{\gamma} R(z, s) \dot{R}(z, s) d z,
$$

where $R(z, s)=(H(s)-z)^{-1}$ and $\gamma$ is a complex contour encircling the part of the spectrum of $H(s)$ lying below $\mu$ and ${ }^{\circ}=\partial / \partial s$. Equation (10) is the 1-particle density matrix which has evolved from that of the Fermi sea, $P_{0}\left(s_{0}\right)$, after a gentle start of the pump. In fact such a start may be obtained from (1) by means of a smooth substitution $s^{\prime} \mapsto s$ with $s^{\prime} \mapsto s_{0},\left(s \leq s_{0}\right)$, and $s^{\prime}=s,\left(s^{\prime}\right.$ large $)$. Then, in the new variable, $P_{1}\left(s_{0}\right)=0$ by $(11)$.

The current across a fiducial point $x=x_{0}$ is the rate of change of the charge contained in $x>x_{0}$ and hence given by the operator $I=\mathrm{i}\left[H(s), \theta\left(x-x_{0}\right)\right]$, which is independent 
of $s$. The charge transported in a cycle (of duration $2 \pi \varepsilon^{-1}$ ) is, in expectation value and in the adiabatic limit, given as

$$
\left\langle Q_{T}\right\rangle:=\oint \operatorname{Tr}\left(I P_{1}(s)\right) d s
$$

because of $d t=\varepsilon^{-1} d s$, with $\operatorname{Tr}$ denoting the trace on $L^{2}\left(\mathbb{R}_{x}, \mathbb{C}^{n}\right)$. This definition rests on the fact that the leading contribution from persistent currents, $\varepsilon^{-1} \oint \operatorname{Tr}\left(I P_{0}(s)\right) d s$, which is potentially divergent in the limit, actually vanishes. If $V$ were real, this would follow trivially from time reversal invariance; however our hypothesis does not imply this, except for $n=1$, and we shall argue otherwise.

The result of [19], generalized as described in the Introduction, is part (ii) of the following theorem.

Theorem 1. Assume (9). Then

i)

$$
\operatorname{Tr}\left(I P_{0}(s)\right)=0
$$

ii)

$$
\left\langle Q_{T}\right\rangle=\frac{\mathrm{i}}{2 \pi} \oint_{\gamma} d z \oint_{S^{1}} d s \operatorname{tr}\left(W\left(\frac{\partial \tilde{\psi}_{-}}{\partial z}, \frac{\partial \psi_{+}}{\partial s} ; x_{0}\right)-W\left(\frac{\partial \tilde{\psi}_{-}}{\partial s}, \frac{\partial \psi_{+}}{\partial z} ; x_{0}\right)\right)
$$

where $\operatorname{tr}$ denotes the matrix trace and the solutions $\psi_{+} \in S_{(z, s)}^{+}, \tilde{\psi}_{-} \in \tilde{S}_{(z, s)}^{-}$satisfying (8) are locally smooth in $(z, s)$. Except for these conditions, the trace is independent of $\psi_{+}, \tilde{\psi}_{-}$, and the integral is of $x_{0}$, too. Moreover, the r.h.s. is the first Chern number of a bundle described in Sect. 3.

We next present the scattering description [7] of charge transport. Consider again the Hamiltonian (1), but now with $V$ of compact support in $x$. As a result, (9) fails:

$$
\mu \in \sigma(H(s))
$$

for all $s$. We may thus introduce the scattering matrix $S(s)$ at Fermi energy $\mu>0$,

$$
S(s)=\left(\begin{array}{ll}
R & T^{\prime} \\
T & R^{\prime}
\end{array}\right)
$$

where the blocks are $n \times n$ matrices determined by the asymptotic behavior of solutions of (2) with $z=\mu$. More precisely, $R$ and $T$ are defined in terms of a plane wave incident from the left,

$$
\psi(x)= \begin{cases}1 \mathrm{e}^{\mathrm{i} k x}+R \mathrm{e}^{-\mathrm{i} k x}, & (x<-r), \\ T \mathrm{e}^{\mathrm{i} k x}, & (x>r),\end{cases}
$$

with $r>0$ large enough and $k=\sqrt{\mu}$. Similarly $R^{\prime}$ and $T^{\prime}$ are defined in terms of a wave incident from the right. 
The charge emitted from all channels of the left lead together, in a cycle and in the adiabatic limit, is [7]

$$
\left\langle Q_{B P T}\right\rangle=\frac{1}{2 \pi \mathrm{i}} \oint \operatorname{tr}\left((d S) S^{*} P\right),
$$

where $d S=(d S / d s) d s$ and $P=\left(\begin{array}{ll}1 & 0 \\ 0 & 0\end{array}\right)$ is the projection onto the left channels. For the same situation the variance is $[1,11]$

$$
\left\langle\left\langle Q_{B P T}^{2}\right\rangle\right\rangle=\frac{1}{(2 \pi)^{2}} \int_{-\infty}^{\infty} d s \oint d s^{\prime} \frac{\operatorname{tr}\left[\left(S^{*}(s) P S(s)-S^{*}\left(s^{\prime}\right) P S\left(s^{\prime}\right)\right)^{2}\right]}{\sin ^{2}\left(s-s^{\prime}\right)} .
$$

In general, and in contrast to (13), $\left\langle Q_{B P T}\right\rangle$ is not an integer. However, $\left\langle\left\langle Q_{B P T}^{2}\right\rangle\right\rangle$ vanishes iff the time dependence of $S$ is of the form

$$
S(s)=\left(\begin{array}{cc}
U_{1}(s) & 0 \\
0 & U_{2}(s)
\end{array}\right) S_{0}
$$

with $U_{j}(s)(j=1,2)$ and $S_{0}$ unitary matrices of order $n$, resp. $2 n$. In this case $\left\langle Q_{B P T}\right\rangle$ is an integer,

$$
\left\langle Q_{B P T}\right\rangle=\frac{1}{2 \pi \mathrm{i}} \oint \operatorname{tr}\left(\left(d U_{1}\right) U_{1}^{*}\right)=\frac{1}{2 \pi \mathrm{i}} \oint d \log \operatorname{det} U_{1},
$$

given as the winding number of $\operatorname{det} U_{1}$.

We do not give here the definition of $\left\langle Q_{B P T}\right\rangle$ which makes (16) a theorem [2]. Rather we focus on the relation between Eqs. (13) and (16). To this end we truncate the potential to a finite interval, $V(x, s) \chi_{[0, L]}(x)$, and denote its scattering matrix by $S_{L}(s)$. In the limit $L \rightarrow \infty$ the original physical situation is recovered and the two approaches agree, as stated in the following result.

Theorem 2. Assume (9) for the infinite system.

i) The scattering matrix $S_{L}(s)$ at Fermi energy $\mu$ has a limit of the form

$$
\lim _{L \rightarrow \infty} S_{L}(s)=\left(\begin{array}{cc}
R(s) & 0 \\
0 & R^{\prime}(s)
\end{array}\right) .
$$

In particular, the condition (17) for quantization of $\left\langle Q_{B P T}\right\rangle$ is attained in the limit. ii) The winding number of det $R(s)$ equals the Chern number on the r.h.s of Eq. (13). In physical terms,

$$
\left\langle Q_{B P T}\right\rangle=\left\langle Q_{T}\right\rangle
$$

We conclude this section by summarizing the idea of the proof of (19). We may assume that the contour $\gamma$ in Eqs. $(11,13)$ crosses the real axis just twice, once below the spectrum and once at Fermi energy $\mu$. The torus of integration in (13), which is denoted by $\mathbb{T}=\gamma \times S^{1}$, is the base space of a bundle which will admit a global section except at isolated points along the line $\{\mu\} \times S^{1} \subset \mathbb{T}$. Using Stokes' theorem its Chern number can be expressed in terms of solutions of the Schrödinger equation at Fermi energy and, in turn, of the scattering matrix (18). The main steps are given in more detail in the following lemma. There the r.h.s. of Eq. (13) is denoted by $C$, and $x_{0}$ is fixed. The orientation of the torus is the natural one, $d \gamma \wedge d s$. 
Lemma 3. i) Any point $\left(z_{*}, s_{*}\right) \in \mathbb{T}$, where det $\psi_{+}\left(x_{0}\right)=0$ for some (and hence all) $\psi_{+} \in S_{\left(z_{*}, s_{*}\right)}^{+}$has $z_{*}=\mu$. For a dense set of potentials $V=V^{*}$, the points $s_{*}$ are isolated in $S^{1}$ and 0 is a simple eigenvalue of $\psi_{+}\left(x_{0}\right)$; moreover,

$$
\operatorname{det} \psi_{+}^{\prime}\left(x_{0}\right) \neq 0 \text {. }
$$

Density is meant w.r.t. the topology of the class of potentials specified below (1).

ii) Let $\psi_{(z, s)} \in S_{(z, s)}^{+}$be a section defined in a neighborhood in $\mathbb{C} \times S^{1} \supset \mathbb{T}$ of any of the above points $\left(z_{*}=\mu, s_{*}\right)$, which is analytic in $z$. Then the family of matrices $L(z, s)=\psi_{(\bar{z}, s)}^{\prime}\left(x_{0}\right)^{*} \psi_{(z, s)}\left(x_{0}\right)$ has the reflection property

$$
L(z, s)=L(\bar{z}, s)^{*} .
$$

Its eigenvalues are real for real $z$. There is a single eigenvalue branch $\lambda(z, s)$ vanishing to first order at $\left(\mu, s_{*}\right)$. Its winding number there is

$$
w_{s_{*}}=-\left.\operatorname{sgn}\left(\frac{\partial \lambda}{\partial z} \frac{\partial \lambda}{\partial s}\right)\right|_{\left(z=\mu, s=s_{*}\right)} .
$$

iii)

$$
C=-\sum_{s_{*}} w_{s_{*}}
$$

iv) At any of the points $\left(\mu, s_{*}\right)$ we have

$$
\frac{\partial \lambda}{\partial z}<0
$$

v) The unitary matrix $R(s)$ has eigenvalue -1 iff det $\psi_{\mu, s}(0)=0$. More precisely, as $s$ increases past $s_{*}$, an eigenvalue of $R$ crosses -1 counterclockwise if

$$
\left.\frac{\partial \lambda}{\partial s}\right|_{\left(z=\mu, s=s^{*}\right)}<0 .
$$

As a result, $C=-\left.\sum_{s_{*}} \operatorname{sgn}(\partial \lambda / \partial s)\right|_{\left(z=\mu, s=s_{*}\right)}$ is the number of eigenvalue crossings of $R(s)$ past -1 , i.e., the winding number of det $R$. Actually the equality is first established if the conditions on the potential of part (i) are satisfied, but the conclusion, Eq. (19), extends by density.

\section{A Fiber Bundle}

We describe the bundle $P$ and the connection underlying Eq. (13). Let $\mathcal{C}=C^{1}\left(\mathbb{R}, M_{n}(\mathbb{C})\right)$ be the space of matrix valued $C^{1}$-functions on $\mathbb{R}$. Let $\pi: P \rightarrow \mathbb{T}$ be the subbundle of $\mathbb{T} \times \mathcal{C}$ with base $\mathbb{T}=\gamma \times S^{1}$ and fibers $S_{(z, s)}^{+} \subset \mathcal{C}:$

$$
P=\left\{((z, s), \psi) \in \mathbb{T} \times \mathcal{C} \mid \psi \in S_{(z, s)}^{+}\right\} .
$$

It is a principal bundle w.r.t. the right action (7) of $\operatorname{GL}(n)$. This includes that $\mathrm{GL}(n)$ is its structure group. Indeed, for any sufficiently small open set $U \subset \mathbb{T}$ there is $x \in \mathbb{R}$ with

$$
\operatorname{det} \psi_{+}(x) \neq 0
$$


for all $\psi_{+} \in S_{(z, s)}^{+}$and $(z, s) \in U$, see Lemma 4 below. This provides a local trivialization $\phi$ with

$$
\phi^{-1}: \pi^{-1}(U) \rightarrow U \times \operatorname{GL}(n), \quad \psi_{+} \mapsto\left(z, s, \psi_{+}(x)\right) .
$$

The transition function $\phi_{2}^{-1} \circ \phi_{1}: \operatorname{GL}(n) \rightarrow \mathrm{GL}(n)$ is multiplication from the left by the matrix $\psi_{+}\left(x_{2}\right) \psi_{+}\left(x_{1}\right)^{-1}$, which is clearly independent of $\psi_{+} \in S_{(z, s)}^{+}$and belongs to GL $(n)$.

We will give an explicit expression for the Chern number $C$ of $P$, which differs somewhat from that used in [19]. We recall that

$$
C=\frac{\mathrm{i}}{2 \pi} \int_{\mathbb{T}} \operatorname{tr} \mathcal{F}
$$

where $\mathcal{F}=D \mathcal{A}$ is the curvature of any connection $\mathcal{A}$ on $P$. We recall that $\operatorname{tr} \mathcal{F}$ defines a 2 -form on $\mathbb{T}$, and not just on $P$; for any two connections, $\mathcal{A}$ and $\mathcal{A}^{\prime}$, the same is true for the 1 -form $\operatorname{tr}\left(\mathcal{A}-\mathcal{A}^{\prime}\right)$, whence $C$ is independent of the choice of connection. We consider connections of the following form. Let $B: \mathcal{C} \times \mathcal{C} \rightarrow M_{n}(\mathbb{C})$ be a bilinear form on $\mathcal{C}$ satisfying

$$
\begin{aligned}
& B(\tilde{\psi}, \psi T)=B(\tilde{\psi}, \psi) T, \\
& B(T \tilde{\psi}, \psi)=T B(\tilde{\psi}, \psi)
\end{aligned}
$$

$(\tilde{\psi}, \psi \in \mathcal{C}, T \in \operatorname{GL}(n))$. Moreover we assume that its restriction

$$
B: \tilde{S}_{(z, s)}^{-} \times S_{(z, s)}^{+} \rightarrow \operatorname{GL}(n)
$$

takes values $B\left(\tilde{\psi}_{-}, \psi_{+}\right)$in the regular matrices (as shown below, an example is (6)). We may then consider the $\mathrm{gl}(n)$-valued 1 -form on $P$

$$
\mathcal{A}_{\psi_{+}}\left(\delta \psi_{+}\right)=B\left(\tilde{\psi}_{-}, \psi_{+}\right)^{-1} B\left(\tilde{\psi}_{-}, \delta \psi_{+}\right), \quad\left(\delta \psi_{+} \in T P\right),
$$

which is well-defined being independent of the choice of $\tilde{\psi}_{-} \in \tilde{S}_{(z, s)}^{-}$by (24). It is a connection on $P$ since it enjoys the defining properties

$$
\begin{aligned}
\mathcal{A}_{\psi_{+}}\left(\psi_{+} t\right) & =t, \quad(t \in \operatorname{gl}(n)), \\
\mathcal{A}_{\psi_{+} T}\left(\delta \psi_{+} T\right) & =T^{-1} \mathcal{A}_{\psi_{+}}\left(\delta \psi_{+}\right) T, \quad(T \in \mathrm{GL}(n))
\end{aligned}
$$

by (23). Given $\psi_{+} \in S_{(z, s)}^{+}$there is a unique $\tilde{\psi}_{-} \in \tilde{S}_{(z, s)}^{-}$such that $B\left(\tilde{\psi}_{-}, \psi_{+}\right)=1$, as can again be seen from (24). Then $\mathcal{A}=B\left(\tilde{\psi}_{-}, \delta \psi_{+}\right)$and the trace of its curvature is

$$
\operatorname{tr} \mathcal{F}=\operatorname{tr}\left(B\left(\frac{\partial \tilde{\psi}_{-}}{\partial z}, \frac{\partial \psi_{+}}{\partial s}\right)-B\left(\frac{\partial \tilde{\psi}_{-}}{\partial s}, \frac{\partial \psi_{+}}{\partial z}\right)\right) d z \wedge d s .
$$

We will use the bilinear

$$
B(\tilde{\psi}, \psi)=W(\tilde{\psi}, \psi ; x)=\tilde{\psi}(x) \psi^{\prime}(x)-\tilde{\psi}^{\prime}(x) \psi(x),
$$

whose restriction (25) is seen to be independent of $x$ (though $\mathcal{A}$ may not be); then (22) coincides with the r.h.s. of (13), as announced in Theorem 1. It remains to verify 
$B\left(\tilde{\psi}_{-}, \psi_{+}\right) \in \operatorname{GL}(n)$. Any column vector solution $\varphi(x)$ of (2) is determined by $\varphi(0)$, $\varphi^{\prime}(0) \in \mathbb{C}^{n}$, similarly for any row vector $\tilde{\varphi}(x)$ solving $(5)$. Their Wronskian

$$
W(\tilde{\varphi}, \varphi)=\tilde{\varphi}(0) \varphi^{\prime}(0)-\tilde{\varphi}^{\prime}(0) \varphi(0),
$$

which now takes values in $\mathbb{C}$, clearly defines a non-degenerate bilinear form on $\mathbb{C}^{2 n}$. Given $\psi_{ \pm} \in S_{(z, s)}^{ \pm}$, any solution $\varphi$ can be expressed as

$$
\varphi(x)=\psi_{+}(x) a_{+}+\psi_{-}(x) a_{-}
$$

with $a_{ \pm} \in \mathbb{C}^{n}$, and $\varphi \equiv 0$ iff $a_{ \pm}=0$; similarly for $\tilde{\varphi}(x)=b_{+} \tilde{\psi}_{+}(x)+b_{-} \tilde{\psi}_{-}(x)$. In terms of the coefficients $\left(b_{+}, b_{-}\right),\left(a_{+}, a_{-}\right)$, the bilinear form (26) is given by the matrix

$$
\left(\begin{array}{cc}
0 & W\left(\tilde{\psi}_{+}, \psi_{-}\right) \\
W\left(\tilde{\psi}_{-}, \psi_{+}\right) & 0
\end{array}\right),
$$

since

$$
W\left(\tilde{\psi}_{ \pm}, \psi_{ \pm}\right)=\lim _{x \rightarrow \pm \infty} W\left(\tilde{\psi}_{ \pm}, \psi_{ \pm} ; x\right)=0
$$

Hence $W\left(\tilde{\psi}_{-}, \psi_{+}\right)$is regular.

Remark. In [19] (and later in [10]) the case of a potential $V(x)$ of period $L$ was considered. In the case $n=1$ the bilinear used there was

$$
B(\tilde{\psi}, \psi)=\int_{0}^{L} d x \tilde{\psi}(x) \psi(x) .
$$

Non-degeneracy of (25) amounts to $\int_{0}^{L} d x \psi_{-}(x) \psi_{+}(x) \neq 0$, where $\psi_{-} \in \tilde{S}_{(z, s)}^{-}=$ $S_{(z, s)}^{-}, \psi_{+} \in S_{(z, s)}^{+}$are unique up to non-zero multiples.

\section{Proofs}

Here we prove Theorems 1 and 2 stated in Sect. 2. First however we should dwell on a little point of precision: The current, informally given as

$$
I=\mathrm{i}[H, \theta(x)]=-\mathrm{i}\left\{\frac{d}{d x}, \delta(x)\right\},
$$

is not a well-defined operator on Hilbert space. (We suppressed $s$ from the notation and set $x_{0}=0$.) Instead, it should be understood as the map $D(H) \rightarrow D(H)^{*}$,

$$
I=\mathrm{i}\left(\gamma_{1}^{*} \gamma_{0}-\gamma_{0}^{*} \gamma_{1}\right)
$$

where $\gamma_{0}, \gamma_{1}: D(H) \rightarrow \mathbb{C}^{n}$ with $\gamma_{0} \psi=\psi(0), \gamma_{1} \psi=\psi^{\prime}(0)$. Then (29) is replaced by

$$
\mathrm{i}[R(z), \theta(x)]=-R(z) I R(z),
$$

which can be verified first as a quadratic form. This operator is of trace class because $\left(p^{2}+1\right)^{-1} \gamma_{i}^{*} \gamma_{i}\left(p^{2}+1\right)^{-1}$ is.

Given an operator $K: D(H)^{*} \rightarrow D(H)$ one may, pretending cyclicity, take

$$
\operatorname{Tr}(I K):=\mathrm{i} \operatorname{tr}\left(\gamma_{0} K \gamma_{1}^{*}-\gamma_{1} K \gamma_{0}^{*}\right)
$$


as a definition. In fact, this is the trace of the finite rank operator $I K$ on the Banach space $D(H)^{*}$, see e.g. [18], Eq. (10.2). It yields

$$
\operatorname{Tr}(I K):=\operatorname{tr}\left(-\mathrm{i} \partial_{1} K(0,0)+\mathrm{i} \partial_{2} K(0,0)\right)
$$

where $K(x, y)$ is the integral kernel of $K$ and $\partial_{1}$ and $\partial_{2}$ indicate a derivative w.r.t. the first, resp. second argument. As a further motivation we note that expectation values of the current are naturally written as $\operatorname{Tr}\left(P_{0} I P_{0}\right)$ and $\operatorname{Tr}\left(P_{0} I P_{1}+P_{1} I P_{0}\right)$ in zeroth and first order in $\varepsilon$. Then

$$
\operatorname{Tr}\left(P_{0} I P_{0}\right)=\mathrm{i} \operatorname{Tr}\left(P_{0}\left(\gamma_{1}^{*} \gamma_{0}-\gamma_{0}^{*} \gamma_{1}\right) P_{0}\right)=\mathrm{i} \operatorname{tr}\left(\gamma_{0} P_{0} \gamma_{1}^{*}-\gamma_{1} P_{0} \gamma_{0}^{*}\right)
$$

where cyclicity is now justified since $\gamma_{i} P_{0}$ is Hilbert-Schmidt; also, $P_{0}^{2}=P_{0}$ was used. Similarly,

$$
\operatorname{Tr}\left(P_{0} I P_{1}+P_{1} I P_{0}\right)=\mathrm{i} \operatorname{tr}\left(\gamma_{0} P_{1} \gamma_{1}^{*}-\gamma_{1} P_{1} \gamma_{0}^{*}\right)
$$

by $P_{0} P_{1}+P_{1} P_{0}=P_{1}$.

Proof of Theorem 1. i) The projection $P_{0}$ has the integral representation $P_{0}=$ $-(2 \pi \mathrm{i})^{-1} \oint_{\gamma} R(z) d z$. Since $\oint_{\gamma} R(z)^{2} d z=0$ we may replace $R(z)$ therein by $R(z)-$ $R(z)^{2} H=-z R(z)^{2}$ :

$$
P_{0}=\frac{1}{2 \pi \mathrm{i}} \oint_{\gamma} z R(z)^{2} d z
$$

We then have, by $(32,30)$,

$$
\begin{aligned}
\operatorname{Tr}\left(P_{0} I P_{0}\right) & =\frac{1}{2 \pi} \oint_{\gamma} z \operatorname{tr}\left(\gamma_{0} R(z)^{2} \gamma_{1}^{*}-\gamma_{1} R(z)^{2} \gamma_{0}^{*}\right) d z \\
& =\frac{1}{2 \pi} \oint_{\gamma} z \operatorname{Tr}\left(R(z)\left(\gamma_{1}^{*} \gamma_{0}-\gamma_{0}^{*} \gamma_{1}\right) R(z)\right) d z \\
& =-\frac{1}{2 \pi} \oint_{\gamma} z \operatorname{Tr}([R(z), \theta(x)]) d z
\end{aligned}
$$

and, by $z R(z)=H R(z)-1$, also $\operatorname{Tr}\left(P_{0} I P_{0}\right)=\mathrm{i} \operatorname{Tr}\left[H P_{0}, \theta\right]$. As the stationarity of $P_{0}$ suggests, the current is independent of $x_{0}$. In fact, upon replacing $\theta(x)$ by $\tilde{\theta}(x)=\theta(x-$ $\left.x_{0}\right)-\theta(x)$ both terms in $\operatorname{Tr}\left(\left(H P_{0}\right) \tilde{\theta}-\tilde{\theta}\left(H P_{0}\right)\right)$ are separately trace class, whence the trace vanishes ([18], Cor. 3.8). We next turn to (33): The commutator $A=[R(z), \theta(x)]$ has integral kernel $A(x, y)=G(x, y)(\theta(y)-\theta(x))$, where $G\left(x, x^{\prime}\right)=R(z)\left(x, x^{\prime}\right)$ is the Green function. By the stated independence we may average over $x_{0}$ instead of setting it to 0 , thus effectively smoothing $\theta$. We will see in $(35,37)$ below that $G(x, y)$ is continuous. Thus $A(x, x)=0$, implying $\operatorname{Tr}\left(P_{0} I P_{0}\right)=0$. Alternatively the conclusion may be reached without smoothing by resorting to Brislawn's theorem ([18], Theorem A.2), according to which $\operatorname{Tr} A=\int d x \tilde{A}(x, x)$, where $\tilde{A}(x, y)$ is the Lebesgue value of $A(x, y)$. Here, $\tilde{A}(x, x)=0$. 
ii) By applying (31) to $K=R(z, s) \dot{R}(z, s)$ in $(12,11)$ we obtain for the transported charge

$$
\left\langle Q_{T}\right\rangle=\frac{\mathrm{i}}{2 \pi} \oint d s \oint_{\gamma} d z \int d x \operatorname{tr}\left(\partial_{1} G(0, x) \dot{G}(x, 0)-G(0, x) \partial_{2} \dot{G}(x, 0)\right) .
$$

We claim that the Green function can be expressed as

$$
G\left(x, x^{\prime}\right)=-\theta\left(x-x^{\prime}\right) \psi_{+}(x) \tilde{\psi}_{-}\left(x^{\prime}\right)-\theta\left(x^{\prime}-x\right) \psi_{-}(x) \tilde{\psi}_{+}\left(x^{\prime}\right),
$$

where we complemented the locally smooth choice of $\psi_{+} \in S_{(z, s)}^{+}, \tilde{\psi}_{-} \in \tilde{S}_{(z, s)}^{-}$satisfying (8) by that of a pair $\tilde{\psi}_{+} \in \tilde{S}_{(z, s)}^{+}, \psi_{-} \in S_{(z, s)}^{-}$with

$$
W\left(\tilde{\psi}_{+}, \psi_{-}\right)=-1 .
$$

Indeed, because of $(8,36)$ and of $(28)$ the general column solution $(27)$ has coefficients

$$
a_{ \pm}= \pm W\left(\tilde{\psi}_{\mp}, \varphi\right)= \pm \tilde{\psi}_{\mp}(y) \varphi^{\prime}(y) \mp \tilde{\psi}_{ \pm}^{\prime}(y) \varphi(y) .
$$

By inserting this in (27) and in its derivative w.r.t. $x$, and by setting $y=x$, we conclude from the arbitrariness of $\varphi(x)$ and $\varphi^{\prime}(x)$ that

$$
\begin{aligned}
& \psi_{+}(x) \tilde{\psi}_{-}(x)-\psi_{-}(x) \tilde{\psi}_{+}(x)=0, \\
& \psi_{+}(x) \tilde{\psi}_{-}^{\prime}(x)-\psi_{-}(x) \tilde{\psi}_{+}^{\prime}(x)=-1, \\
& \psi_{+}^{\prime}(x) \tilde{\psi}_{-}(x)-\psi_{-}^{\prime}(x) \tilde{\psi}_{+}(x)=1 .
\end{aligned}
$$

By means of these relations one verifies that $G$, as given by the r.h.s. of (35), satisfies

$$
\left(-\frac{d^{2}}{d x^{2}}+V(x)-z\right) G\left(x, x^{\prime}\right)=\delta\left(x-x^{\prime}\right) 1
$$

together with $G\left(x, x^{\prime}\right) \rightarrow 0,(|x| \rightarrow \infty)$, which exhibits it as the Green function. We then apply (35) in Eq. (34): For $x \geq 0$ the integrand is

$$
\begin{aligned}
\operatorname{tr} & \left(\partial_{1} G(0, x) \dot{G}(x, 0)-G(0, x) \partial_{2} \dot{G}(x, 0)\right) \\
= & \operatorname{tr}\left(\psi_{-}^{\prime}(0) \tilde{\psi}_{+}(x)\left(\dot{\psi}_{+}(x) \tilde{\psi}_{-}(0)+\psi_{+}(x) \dot{\tilde{\psi}}_{-}(0)\right)\right. \\
& \left.-\psi_{-}(0) \tilde{\psi}_{+}(x)\left(\dot{\psi}_{+}(x) \tilde{\psi}_{-}^{\prime}(0)+\psi_{+}(x) \dot{\tilde{\psi}}_{-}^{\prime}(0)\right)\right)=\operatorname{tr}\left(W\left(\dot{\tilde{\psi}}_{-}, \psi_{-}\right) \tilde{\psi}_{+}(x) \psi_{+}(x)\right),
\end{aligned}
$$

where we used cyclicity of the trace and (28). Here and henceforth the Wronskian is evaluated at $x=0$, unless otherwise stated. Together with a similar computation for $x \leq 0$ we obtain

$$
\begin{aligned}
\left\langle Q_{T}\right\rangle= & \frac{\mathrm{i}}{2 \pi} \oint d s \oint_{\gamma} d z \\
& \times \operatorname{tr}\left(W\left(\dot{\tilde{\psi}}_{-}, \psi_{-}\right) \int_{0}^{\infty} d x \tilde{\psi}_{+}(x) \psi_{+}(x)+W\left(\dot{\tilde{\psi}}_{+}, \psi_{+}\right) \int_{-\infty}^{0} d x \tilde{\psi}_{-}(x) \psi_{-}(x)\right) .
\end{aligned}
$$


We maintain that the same expression is obtained from a computation of $C$, the r.h.s. of (13). That calls for one of $\partial \psi_{+} / \partial z, \partial \tilde{\psi}_{-} / \partial z$. Differentiating (2) w.r.t. $z$ we obtain

$$
\left(-\frac{d^{2}}{d x^{2}}+V(x, s)-z\right) \frac{\partial \psi_{+}}{\partial z}=\psi_{+}
$$

whose general solution with $\partial \psi_{+} / \partial z \rightarrow 0,(x \rightarrow \infty)$ is

$$
\frac{\partial \psi_{+}}{\partial z}(x)=\psi_{+}(x) F_{+}(x)-\psi_{-}(x) \int_{x}^{\infty} \tilde{\psi}_{+}\left(x^{\prime}\right) \psi_{+}\left(x^{\prime}\right) d x^{\prime},
$$

where $F_{+}^{\prime}(x)=d F_{+} / d x=-\tilde{\psi}_{-}(x) \psi_{+}(x)$. Hence $F_{+}$is determined up to an additive constant, which reflects the gauge freedom (7) of $\psi_{+}$. Equation (39) is verified by twice differentiating it w.r.t. $x$, the first derivative being

$$
\frac{\partial \psi_{+}^{\prime}}{\partial z}(x)=\psi_{+}^{\prime}(x) F_{+}(x)-\psi_{-}^{\prime}(x) \int_{x}^{\infty} \tilde{\psi}_{+}\left(x^{\prime}\right) \psi_{+}\left(x^{\prime}\right) d x^{\prime},
$$

by using (37). In the same way we find

$$
\frac{\partial \tilde{\psi}_{-}}{\partial z}(x)=F_{-}(x) \tilde{\psi}_{-}(x)-\left(\int_{-\infty}^{x} \tilde{\psi}_{-}\left(x^{\prime}\right) \psi_{-}\left(x^{\prime}\right) d x^{\prime}\right) \tilde{\psi}_{+}(x),
$$

with $F_{-}^{\prime}=-F_{+}^{\prime}$. The arbitrariness of $F_{ \pm}$is constrained by (8), which implies

$$
F_{+}+F_{-}=0 \text {. }
$$

This is seen by differentiating the constraint w.r.t. $z$ and by using

$$
\begin{aligned}
W\left(\tilde{\psi}_{-}, \frac{\partial \psi_{+}}{\partial z} ; x\right)= & W\left(\tilde{\psi}_{-}, \psi_{+} ; x\right) F_{+}(x)-W\left(\tilde{\psi}_{-}, \psi_{-} ; x\right) \\
& \times \int_{x}^{\infty} \tilde{\psi}_{+}(x) \psi_{+}(x) d x=F_{+}(x), W\left(\frac{\partial \tilde{\psi}_{-}}{\partial z}, \psi_{+} ; x\right)=F_{-}(x) .
\end{aligned}
$$

Similarly, differentiating the constraint w.r.t. $s$ yields

$$
W\left(\dot{\tilde{\psi}}_{-}, \psi_{+} ; x\right)+W\left(\tilde{\psi}_{-}, \dot{\psi}_{+} ; x\right)=0 .
$$

We are now in position to compute $C$ and in particular

$$
\begin{aligned}
W\left(\frac{\partial \tilde{\psi}_{-}}{\partial s}, \frac{\partial \psi_{+}}{\partial z}\right)= & \dot{\tilde{\psi}}_{-}(0)\left(\psi_{+}^{\prime}(0) F_{+}(0)-\psi_{-}^{\prime}(0) \int_{0}^{\infty} \tilde{\psi}_{+}(x) \psi_{+}(x) d x\right) \\
& -\dot{\tilde{\psi}}_{-}^{\prime}(0)\left(\psi_{+}(0) F_{+}(0)-\psi_{-}(0) \int_{0}^{\infty} \tilde{\psi}_{+}(x) \psi_{+}(x) d x\right) \\
= & W\left(\dot{\tilde{\psi}}_{-}, \psi_{+}\right) F_{+}(0)-W\left(\dot{\tilde{\psi}}_{-}, \psi_{-}\right) \int_{0}^{\infty} \tilde{\psi}_{+}(x) \psi_{+}(x) d x, \\
W\left(\frac{\partial \tilde{\psi}_{-}}{\partial z}, \frac{\partial \psi_{+}}{\partial s}\right)= & F_{-}(0) W\left(\tilde{\psi}_{-}, \dot{\psi}_{+}\right)-\left(\int_{-\infty}^{0} \tilde{\psi}_{-}(x) \psi_{-}(x) d x\right) W\left(\tilde{\psi}_{+}, \dot{\psi}_{+}\right) .
\end{aligned}
$$

Taking the trace of difference of the two expressions, the first terms on the r.h.s. cancel because of $(40,41)$. The result is that $C$ agrees with the r.h.s. of (38).

The stated independence of the trace follows from its cyclicity by joining the left and right actions (7) in such a way as to preserve (8); that of the integral is explained after Eq. (22). 
Proof of Theorem 2. i) We recall that the scattering matrix $S_{L}=\left(\begin{array}{ll}R_{L} & T_{L}^{\prime} \\ T_{L} & R_{L}^{\prime}\end{array}\right)$ is that of the potential truncated to the interval $[0, L]$. The left incident solution of (2) is given by the expressions (15) in the intervals $x \leq 0$, resp. $x \geq L$. Its adjoint is a solution of (5) since $z=\mu$ is real. By the constancy of the Wronskian,

$$
W\left(1 \mathrm{e}^{-\mathrm{i} k x}+R_{L}^{*} \mathrm{e}^{\mathrm{i} k x}, \psi_{ \pm} ; x=0\right)=W\left(T_{L}^{*} \mathrm{e}^{-\mathrm{i} k x}, \psi_{ \pm} ; x=L\right),
$$

and by $W\left(1 \mathrm{e}^{\mathrm{i} k x}, \psi_{ \pm} ; x\right)=\mathrm{e}^{\mathrm{i} k x}\left(\psi_{ \pm}^{\prime}(x)-\mathrm{i} k \psi_{ \pm}(x)\right)$ we find

$$
\left(\psi_{ \pm}^{\prime}(0)+\mathrm{i} k \psi_{ \pm}(0)\right)+R_{L}^{*}\left(\psi_{ \pm}^{\prime}(0)-\mathrm{i} k \psi_{ \pm}(0)\right)=T_{L}^{*} \mathrm{e}^{-\mathrm{i} k L}\left(\psi_{ \pm}^{\prime}(L)+\mathrm{i} k \psi_{ \pm}(L)\right)
$$

We have that

$$
\begin{array}{r}
\lim _{x \rightarrow+\infty} \psi_{+}^{\prime}(x)+\mathrm{i} k \psi_{+}(x)=0, \\
\lim _{x \rightarrow+\infty}\left(\psi_{-}^{\prime}(x)+\mathrm{i} k \psi_{-}(x)\right)^{-1}=0 .
\end{array}
$$

Indeed, the first limit just repeats the definition (4) and the second may be rephrased to the effect that

$$
A(x):=\left(\psi_{-}^{\prime}(x)+\mathrm{i} k \psi_{-}(x)\right)^{*}\left(\psi_{-}^{\prime}(x)+\mathrm{i} k \psi_{-}(x)\right)
$$

is invertible with $\lim _{x \rightarrow+\infty}\left\|A(x)^{-1}\right\|=0$. We note that

$$
A(x)=\psi_{-}^{\prime}(x)^{*} \psi_{-}^{\prime}(x)+k^{2} \psi_{-}(x)^{*} \psi_{-}(x),
$$

since the cross term is $-\mathrm{i} k W\left(\psi_{-}^{*}, \psi_{-}\right)=0$ by (28). If the claim were false, there would exist a sequence $x \rightarrow \infty$ and $a(x) \in \mathbb{C}^{n},(\|a(x)\|=1)$ such that $\left\|\psi_{-}^{\prime}(x) a(x)\right\|+$ $\left\|\psi_{-}(x) a(x)\right\|$ remains bounded. Together with (43) this however contradicts the fact that $W\left(\psi_{+}^{*}, \psi_{-}\right)$is regular. Having so established (44), we multiply the - version of (42) by $\mathrm{e}^{\mathrm{i} k L}\left(\psi_{-}^{\prime}(L)-\mathrm{i} k \psi_{-}(L)\right)^{-1}$ from the right, while keeping the + version unchanged. As $L \rightarrow+\infty$ the two equations then go over to

$$
\begin{aligned}
\left(\psi_{+}^{\prime}(0)+\mathrm{i} k \psi_{+}(0)\right)+R^{*}\left(\psi_{+}^{\prime}(0)-\mathrm{i} k \psi_{+}(0)\right) & =0, \\
0 & =T^{*},
\end{aligned}
$$

in the sense that the coefficients do. Since the latter system has a unique solution $\left(R^{*}, T^{*}\right)$, it is the limit of $\left(R_{L}^{*}, T_{L}^{*}\right)$.

ii) As indicated at the end of Sect. 2, part (ii) is an immediate consequence of Lemma 3.

As a preliminary to the proof of Lemma 3(i) we state:

Lemma 4. Let $\psi_{+} \in S_{(z, s)}^{+}$and $x \in \mathbb{R}$. Then 0 is an eigenvalue of $\psi_{+}(x)$ iff $z$ is a Dirichlet eigenvalue for $H(s)$ on $[x, \infty)$, including multiplicities. These conditions can occur only for $z \in \mathbb{R}$ and for isolated $x$. 
Proof. Solutions $\varphi=\varphi(x)$ with values in $\mathbb{C}^{n}$ of the differential equation $H(s) \varphi=z \varphi$ are square-integrable at $x=+\infty$ iff $\varphi(x)=\psi_{+}(x) a$ for some $a \in \mathbb{C}^{n}$. Hence the equivalence of the two conditions. They imply $z \in \mathbb{R}$ because the operator $H(s)$ with Dirichlet boundary conditions on $[x, \infty)$ is self-adjoint. To show that $x$ is isolated, we assume $x=0$ without loss and Taylor expand $\psi_{+}(x)$ at $x=0$ up to second order. Using (2) on the second derivative, we so obtain

$$
\begin{aligned}
& \psi_{+}(x)^{*} \psi_{+}(x) \\
& =P^{\perp}\left(\psi_{+}(0)^{*} \psi_{+}(0)+x\left(\psi_{+}^{\prime}(0)^{*} \psi_{+}(0)+\psi_{+}(0)^{*} \psi_{+}^{\prime}(0)\right)+x^{2} \psi_{+}(0)^{*}(V(0)-z) \psi_{+}(0)\right) \\
& \quad \times P^{\perp}+x^{2} \psi_{+}^{\prime}(0)^{*} \psi_{+}^{\prime}(0)+o\left(x^{2}\right), \quad(x \rightarrow 0),
\end{aligned}
$$

where an orthogonal projection $P^{\perp}=1-P$ onto $\left(\operatorname{ker} \psi_{+}(0)\right)^{\perp}$ has been inserted for free as a result of $\psi_{+}(0) P=0$ and of $\psi_{+}^{\prime}(0)^{*} \psi_{+}(0)=\psi_{+}(0)^{*} \psi_{+}^{\prime}(0)$, which follows from (21) for $\bar{z}=z$. For small $x \neq 0$ the two terms are positive semidefinite, with the first one being definite on $\left(\operatorname{ker} \psi_{+}(0)\right)^{\perp}$. Since

$$
\operatorname{ker} \psi_{+}(0) \cap \operatorname{ker} \psi_{+}^{\prime}(0)=\{0\}
$$

by (3), their sum is positive definite on all of $\mathbb{C}^{n}$. Hence $\psi_{+}(x)$ is regular.

Proof of Lemma 3. We keep $x_{0}=0$ throughout the proof.

i) If at $\left(z_{*}, s_{*}\right)$ a matrix $\psi_{+}(0)$ is singular, that remains true under gauge transformations (7). By the previous lemma, $z_{*} \in \gamma$ is real and not below the spectrum of $H\left(s_{*}\right)$. It remains to prove the properties holding true for a dense set of potentials. Eigenvalue curves $f(s)$ of the Dirichlet Hamiltonian $H(s)$ on $[0, \infty)$ are continuously differentiable, even through crossings. By Sard's theorem the set $\left\{\mu^{\prime} \in \mathbb{R} \mid f\left(s_{*}\right)=\mu^{\prime}, f^{\prime}\left(s_{*}\right)=\right.$ 0 for some $\left.s_{*} \in S^{1}\right\}$ has zero measure. Upon adding to $V(x, s)$ an arbitrarily small constant we may assume that $\mu$ is not in that set. In particular, the points $s_{*}$ are isolated, as claimed. We further perturb $V$ by $t W(x, s)$, where $t$ is small and $W=W(x, s)$ is an arbitrary Hermitian matrix from the same class as $V$. To first order in $t$, the splitting of a degenerate Dirichlet eigenvalue $\mu$ of $H\left(s_{*}\right)$ is $\mu+t \tilde{\mu}+o\left(t^{2}\right),(t \rightarrow 0)$, where the $\tilde{\mu}$ are obtained by solving the finite dimensional eigenvalue problem

$$
\begin{aligned}
& P\left(\int_{0}^{\infty} d x \psi_{+}(x)^{*} W\left(x, s_{*}\right) \psi_{+}(x)\right) P a \\
& \quad=\tilde{\mu} P\left(\int_{0}^{\infty} d x \psi_{+}(x)^{*} \psi_{+}(x)\right) P a, \quad\left(a \in \mathbb{C}^{n}\right),
\end{aligned}
$$

and $P$ is again the projection onto $\operatorname{ker} \psi_{+}(0)$. Since $\psi_{+}(x)$ is regular a.e., the matrix in brackets on the 1.h.s. may take arbitrary Hermitian values, while that on the r.h.s. is positive definite on $\mathbb{C}^{n}$; the latter may then be set equal to 1 by means of a gauge transformation. As a result, the eigenvalues $\tilde{\mu}$ are generically distinct and, since $f^{\prime}\left(s_{*}\right) \neq 0$, the points $s_{*}$ split into non-degenerate ones. Moreover, points $s_{*}$ with det $\psi_{+}^{\prime}\left(x_{0}\right)=0$ correspond to Neumann eigenvalues. They are also perturbed and split according to (47), except that $P$ now is the projection onto ker $\psi_{+}^{\prime}(0)$. Because of (46) the coincidence between Dirichlet and Neumann eigenvalues is generically lifted. 
ii) If $\psi_{(z, s)}(x)$ is a solution of $(2)$, then $\psi_{(\bar{z}, s)}(x)^{*}$ is a solution of (5). Hence

$$
L(\bar{z}, s)^{*}-L(z, s)=W\left(\psi_{(\bar{z}, s)}^{*}, \psi_{(z, s)} ; 0\right)=0,
$$

by (28), proving the reflection property. The statement about the eigenvalue branch follows from (i). The winding number can be read off from the linearization

$$
\lambda(z, s)=\left.\frac{\partial \lambda}{\partial z}\right|_{\left(\mu, s_{*}\right)} \cdot(z-\mu)+\left.\frac{\partial \lambda}{\partial s}\right|_{\left(\mu, s_{*}\right)} \cdot\left(s-s_{*}\right)+O\left(|z-\mu|^{2}+\left|s-s_{*}\right|^{2}\right),
$$

where the derivatives are real.

iii) In view of the right action (7) a section $\psi_{+}^{0}:(z, s) \mapsto \psi_{(z, s)}^{0}(x)$ may be defined on all of the torus by $\psi_{(z, s)}^{0}(0)=1$, except for the points $\left(\mu, s_{*}\right)$ of part (i). We use it outside of the union $\cup_{s_{*}} U_{S_{*}}$ of arbitrarily small neighborhoods of those points; inside we use a section $\hat{\psi}_{+}$defined there. Using these local sections, the connection is expressed as a 1 -form on the corresponding patches of the torus, e.g. $\psi_{+}^{0 *} \mathcal{A}$ (with ${ }^{*}$ exceptionally denoting the pull-back), and the trace of the curvature as a 2-form, $\operatorname{tr} D \mathcal{A}=d \operatorname{tr} \psi_{+}^{0 *} \mathcal{A}$. Upon changing the patch we have $\hat{\psi}_{+}=\psi_{+}^{0} T$ with $T=T(z, s) \in \operatorname{GL}(n)$ and hence $\hat{\psi}_{+}^{*} \mathcal{A}=T^{-1}\left(\psi_{+}^{0 *} \mathcal{A}\right) T+T^{-1}(d T)$. So, using Stokes' theorem on (22), we express the Chern number as

$$
C=\frac{\mathrm{i}}{2 \pi} \sum_{s_{*}} \oint_{\partial U_{s_{*}}} \operatorname{tr} \hat{\psi}_{+}^{*} \mathcal{A}-\operatorname{tr} \psi_{+}^{0 *} \mathcal{A}=\frac{\mathrm{i}}{2 \pi} \oint_{\partial U_{s_{*}}} d \log \operatorname{det} T .
$$

We may here replace $T=\hat{\psi}_{(z, s)}(0) \psi_{(z, s)}^{0}(0)^{-1}=\hat{\psi}_{(z, s)}(0)$ by $L(z, s)$, because of $(20)$. In $U_{s_{*}}$ we have $L(z, s)=\lambda(z, s) P(z, s)+\tilde{L}(z, s)$, where $P(z, s)$ is a rank 1 projection and $\tilde{L}(z, s)$ is a regular linear map from $\operatorname{ker} P(z, s)$ to itself. Thus det $L$ can be in turn replaced by $\operatorname{det}(\lambda P)=\lambda$ and the claim follows.

iv) Let $u \in \mathbb{C}^{n}$ be the normalized eigenvector of $L\left(\mu, s_{*}\right)$ with eigenvalue $\lambda\left(\mu, s_{*}\right)=0$. Then

$$
\left.\frac{\partial \lambda}{\partial z}\right|_{\left(\mu, s_{*}\right)}=\left(u,\left.\frac{\partial L}{\partial z}\right|_{\left(\mu, s_{*}\right)} u\right)=\left(u, \psi_{+}^{\prime *} \frac{\partial \psi_{+}}{\partial z} u\right),
$$

since $\psi_{+} u=0$ at $\left(z=\mu, s=s_{*}\right)$. There we may write

$$
\frac{\partial \lambda}{\partial z}=\left(u,\left(\psi_{+}^{* \prime} \frac{\partial \psi_{+}}{\partial z}-\psi_{+}^{*} \frac{\partial^{2} \psi_{+}}{\partial x \partial z}\right) u\right)=-\left(u, W\left(\psi_{+}^{*}, \frac{\partial \psi_{+}}{\partial z} ; x=0\right) u\right) .
$$

On the other hand we have

$$
W\left(\psi_{+}^{*}, \frac{\partial \psi_{+}}{\partial z} ; x\right)=\int_{x}^{\infty} d x^{\prime} \psi_{+}^{*}\left(z, x^{\prime}\right) \psi_{+}\left(z, x^{\prime}\right)>0
$$

which follows by differentiating (6) w.r.t. $x$ and by using (2).

v) The matrix $R$ in (18) is determined by (45) or, after multiplication with $R$,

$$
R\left(\psi_{+}^{\prime}(0)+\mathrm{i} k \psi_{+}(0)\right)+\left(\psi_{+}^{\prime}(0)-\mathrm{i} k \psi_{+}(0)\right)=0 .
$$


This shows that $\psi_{+}(0)$ has eigenvalue 0 iff $R$ has eigenvalue $-1: \psi_{+}(0) u=0$ implies $(R+1) \psi_{+}^{\prime}(0) u=0$; conversely $(R+1) v=0$ implies $R^{*} v=-v$ and then $\psi_{+}^{*}(0) v=0$. Moreover

$$
\dot{R}\left(\psi_{+}^{\prime}(0)+\mathrm{i} k \psi_{+}(0)\right)+R\left(\dot{\psi}_{+}^{\prime}(0)+\mathrm{i} k \dot{\psi}_{+}(0)\right)+\dot{\psi}_{+}^{\prime}(0)-\mathrm{i} k \dot{\psi}_{+}(0)=0 .
$$

We compute the rate at which the eigenvalue crosses -1 as

$$
\dot{Z}=\frac{\left(\psi_{+}^{\prime}(0) u, \dot{R} \psi_{+}^{\prime}(0) u\right)}{\left(\psi_{+}^{\prime}(0) u, \psi_{+}^{\prime}(0) u\right)}
$$

since the eigenprojection of the unitary $R$ is orthogonal. Multiplying (49) with $\psi_{+}^{\prime}(0) u$ from the left and with $u$ from the right we obtain, using $R^{*} \psi_{+}^{\prime}(0) u=-\psi_{+}^{\prime}(0) u$,

$$
\left(\psi_{+}^{\prime}(0) u, \dot{R} \psi_{+}^{\prime}(0) u\right)-2 \mathrm{i} k\left(\psi_{+}^{\prime}(0) u, \dot{\psi}_{+}(0) u\right)=0,
$$

and hence

$$
\dot{Z}\left(\psi_{+}^{\prime}(0) u, \psi_{+}^{\prime}(0) u\right)=2 \mathrm{i} k \frac{\partial \lambda}{\partial s}
$$

\section{A. Adiabatic Evolution}

We consider the usual quantum mechanical, adiabatic setting in the presence of a spectral gap: A family of operators $H(s)$ depending smoothly on $s$ and corresponding spectral projections $P_{0}(s)$ belonging to an interval $I(s)$ whose endpoints lie in the resolvent set $\rho(H(s))$. Let $U_{\varepsilon}\left(s, s_{0}\right)$ be the propagator for the non-autonomous Hamiltonian $H(s)$ with $s=\varepsilon t$. Then

$$
U_{\varepsilon}\left(s, s_{0}\right)\left(P_{0}\left(s_{0}\right)+\varepsilon P_{1}\left(s_{0}\right)\right) U_{\varepsilon}\left(s, s_{0}\right)^{*}=P_{0}(s)+\varepsilon P_{1}(s)+O\left(\varepsilon^{2}\right), \quad(\varepsilon \rightarrow 0)
$$

with $P_{1}(s)$ as given by Eq. (11). This result is implicit in [19]. We give an alternate derivation which does not approximate the continuous spectrum by a quasi-continuum of discrete eigenvalues.

Proof. In Eq. (10) $P_{1}(s)$ is uniquely determined [15] by the conditions

$$
\begin{aligned}
\text { i } \dot{P}_{0}(s) & =\left[H, P_{1}(s)\right], \\
P_{0}(s) P_{1}(s)+P_{1}(s) P_{0}(s) & =P_{1}(s),
\end{aligned}
$$

which are obtained by differentiating the expansion w.r.t. $s$, respectively from the fact that it represents a projection. We omit $s$ from the notation in the rest of the proof. Equation (11) satisfies the first condition because of

$$
\left[H, P_{1}\right]=-\frac{1}{2 \pi} \oint_{\gamma}[H-z, R(z) \dot{R}(z)] d z=-\frac{1}{2 \pi} \oint_{\gamma}\left(\dot{R}(z)+R(z)^{2} \dot{H}\right) d z,
$$


where we expanded the commutator and used $\dot{R}=-R \dot{H} R$. The second contribution vanishes and the first yields the claim by $P_{0}=-(2 \pi \mathrm{i})^{-1} \oint_{\gamma} R(z) d z$. The second condition (50) is equivalent to $P_{0} P_{1} P_{0}=0,\left(1-P_{0}\right) P_{1}\left(1-P_{0}\right)=0$, which are satisfied, too: we rewrite $\dot{R}$ as before and use the spectral representation $P=\int_{I} d P_{\lambda}$ to compute

$$
P_{0} P_{1} P_{0}=\int_{I} \int_{I}\left(d P_{\lambda}\right) \dot{H}\left(d P_{\mu}\right) \oint_{\gamma} d z \frac{1}{(\lambda-z)^{2}(\mu-z)}=0 ;
$$

similarly, $\left(1-P_{0}\right) P_{1}\left(1-P_{0}\right)=0$.

We may add that in [3], Eq. (2.6) and [4], Eq. (2.10a), as well as in [15], Eq. (2.28), the expression

$$
P_{1}(s)=-\frac{1}{2 \pi} \oint_{\gamma(s)} R(z, s)[\dot{P}(s), P(s)] R(z, s) d z
$$

is given. Its equality with (11) can be verified independently of (50).

Acknowledgements. We thank M. Büttiker for drawing our attention to the multi-channel case, and S. Jansen and R. Seiler for discussions. We are grateful to A. Elgart who contributed the above derivation of Eq. (11) in replacement of a longer one.

\section{References}

1. Avron, J.E., Elgart, A., Graf, G.M., Sadun, L.: Transport and dissipation in quantum pumps. J. Stat. Phys. 116, 425-473 (2004)

2. Avron, J.E., Elgart, A., Graf, G.M., Sadun, L., Schnee, K.: Adiabatic charge pumping in open quantum systems. Comm. Pure Appl. Math. 57, 528-561 (2004)

3. Avron, J.E., Seiler, R., Yaffe, L.G.: Adiabatic theorems and applications to the quantum Hall effect. Commun. Math. Phys. 110, 33-49 (1987)

4. Avron, J.E., Seiler, R., Yaffe, L.G.: Erratum: "Adiabatic theorems and applications to the quantum Hall effect”. Commun. Math. Phys. 156, 649-650 (1993)

5. Blumenthal, M.D., Kaestner, B., Li, L., Giblin, S., Janssen, T.J.B.M., Pepper, M., Anderson, D., Jones, G., Ritchie, D.A.: Gigahertz quantized charge pumping. Nat. Phys. 3, 343-347 (2007)

6. Brouwer, P.W.: Scattering approach to parametric pumping. Phys. Rev. B 58, R10135 (1998)

7. Büttiker, M., Thomas, H., Prêtre, A.: Current partition in multiprobe conductors in the presence of slowly oscillating external potentials. Z. Phys. B 94, 133-137 (1994)

8. Chern, C.H., Onoda, S., Murakami, S., Nagaosa, N.: Quantum charge pumping and electric polarization in Anderson insulators. Phys. Rev B 76, 035334 (2007)

9. Clark, S., Gesztesy, F.: On Povzner-Wienholtz-type self-adjointness results for matrix-valued SturmLiouville operators. Proc. Roy. Soc. Edinburgh Sect. A 133, 747-758 (2003)

10. Graf, G.M., Ortelli, G.: Comparison of quantization of charge transport in periodic and open pumps. Phys. Rev. B 77, 033304 (2008)

11. Ivanov, D.A., Lee, H.W., Levitov, L.S.: Coherent states of alternating current. Phys. Rev. B 56, 6839-6850 (1997)

12. Leek, P.J., Buitelaar, M.R., Talyanskii, V.I., Smith, C.G., Anderson, D., Jones, G.A.C., Wei, J., Cobden, D.H.: Phys. Rev. Lett. 95, 256802 (2005)

13. Lesch, M., Malamud, M.: On the deficiency indices and self-adjointness of symmetric Hamiltonian systems. J. Diff. Eqs. 189, 556-615 (2003)

14. Lidskii, V.B.: On the number of solutions with integrable square of differential equations. Dokl. Akad. Nauk SSSR 95, 217-220 (1954) (in Russian)

15. Nenciu, G.: Linear adiabatic theory. Exponential estimates. Commun. Math. Phys. 152, 479-496 (1993)

16. Niu, Q., Thouless, D.J.: Quantised adiabatic charge transport in the presence of substrate disorder and many-body interaction. J. Phys. A 17, 2453-2462 (1984)

17. Shilton, J.M., Talyanskii, V.I., Pepper, M., Ritchie, D.A., Frost, J.E.F., Ford, C.J.B., Smith, C.G., Jones, G.A.C.: J. Phys. C 8, L531-L539 (1996) 
18. Simon, B.: Trace Ideals and Their Applications. 2nd edition, Providence, RI: Amer. Math. Soc., 2005

19. Thouless, D.J.: Quantisation of particle transport. Phys. Rev. B 27, 6083-6087 (1983)

Communicated by M. Aizenman 